\title{
Cellulitis in Patients with Cirrhosis and Edema: An Under-Recognized Complication Currently More Common than Spontaneous Bacterial Peritonitis
}

\author{
Catherine Rongey ${ }^{1}$, Nelson H. Lim² ${ }^{2}$ and Bruce A. Runyon ${ }^{*}, 3$ \\ ${ }^{I}$ Department of Internal Medicine, Division of Gastroenterology and Hepatology, University of Southern California, \\ Los Angeles, CA 90033, USA \\ ${ }^{2}$ Department of Internal Medicine, Division of Gastroenterology, Loma Linda University Medical Center, Loma Linda, \\ CA 92354, USA \\ ${ }^{3}$ Study conducted at Rancho Los Amigos Medical Center, Liver Unit, Department of Internal Medicine, Downey, Cali- \\ fornia 90242, USA
}

\begin{abstract}
Background: Cellulitis is often a recurring infection resulting in hospitalizations. In patients with cirrhosis, gram-negative bacilli may be causative.

Methods: Retrospective chart review of patients admitted to a dedicated Liver Unit in a tertiary referral center. Risk factors, characteristics and antibiotic treatment of patients with cirrhosis and edema complicated by cellulitis were compared to similar patients without cellulitis. We compared the frequency of cellulitis to that of spontaneous bacterial peritonitis in the same cohort. Our study is the largest case series focusing on the factors which predispose patients with cirrhosis to cellulitis.

Results: Of 145 consecutive patients who were admitted to this unit during 1 year's time, $19.3 \%$ were diagnosed with cellulitis compared to $4.1 \%$ diagnosed with spontaneous bacterial peritonitis. Cellulitis recurred in $21.4 \%$ of our patients. Significant risk factors for cellulitis included history of trauma or break in the skin barrier, homelessness, body mass index on admission and subjective degree of ascites and edema. Hospital stay was four days longer in patients with cellulitis, although this finding was not statistically significant. The duration of intravenous antibiotic treatment was 11.9 days.

Conclusions: The incidence and recurrence rates of cellulitis in patients with cirrhosis and edema are very high and appear to be higher than those of spontaneous bacterial peritonitis in the current era. The diagnosis of cellulitis remains a clinical one as laboratory values and body temperature may not reveal the diagnosis and wound cultures are not usually helpful. Optimal antibiotic treatment warrants further delineation as continued intravenous antibiotic treatment may prolong hospitalization.
\end{abstract}

\section{INTRODUCTION}

Although approximately $75 \%$ of all infections in patients with cirrhosis are caused by gram-negative organisms, it is commonly assumed that skin infections are most likely caused by gram-positive organisms [1,2]. Staphylococcus aureus and Streptococcus pyogenes are responsible for the majority of cellulitis cases in patients without cirrhosis $[1$, 2]. Gram-negative infections are considered in perianal cellulitis, immunocompromised patients $[3,4]$ and patients with nephrotic syndrome [5]. There are several case studies [6-10] and one case series [11] of gram-negative cellulitis in patients with cirrhosis. Although penicillins are currently accepted as first-line treatment for group A Streptococcus cellulites [12], broader coverage should probably be considered in resistant cellulitis in patients with cirrhosis. Our study is

*Address correspondence to this author at the Liver Service, Loma Linda University Medical Center, Loma Linda, CA 92354, USA;

E-mail: BRunyon@ahs.llumc.edu the largest case series focusing on the factors that predispose patients with cirrhosis to cellulitis.

\section{METHODS}

A retrospective chart review from September 2000 through September 2001 was performed investigating cellulitis in a population of patients with ascites due to cirrhosis. Cellulitis was defined by the presence of erythema, warmth and swelling. The control population was identified through a chart review of all patients admitted to one team of our liver unit. The control population was defined by ascites due to cirrhosis and without a past medical history of cellulitis. We compared categorical variables between groups using a chi-square analysis. We used the Mann Whitney U test for continuous variables. A p-value less than 0.05 was considered to indicate statistical significance. This study was approved by the institutional review board of Rancho Los Amigos. 


\section{RESULTS}

Sixty-one control patients were identified, but of these 3 had ascites secondary to etiology other than cirrhosis, 7 had cellulitis in the past, 3 had developed spontaneous bacteremia of unknown etiology and 16 charts could not be found. A chart review was performed on the remaining 32 charts.

\section{Etiology of Cirrhosis}

Our cellulitis and control population groups were similar with a non-significant difference found between age, gender, race and etiologies of cirrhosis which included alcohol, nonalcoholic steatohepatitis (NASH), hepatitis B and hepatitis C (Table 1). The population was predominantly male in both groups and alcohol appeared to be the most common etiology of cirrhosis. Sites of infection varied with the most common site being the lower extremity (Table 2 ).

Table 1. Baseline Characteristics of Study Population

\begin{tabular}{|l|c|c|c|}
\hline & Cellulitis & Control & p-Value \\
\hline \hline Age & 48.8 & 46.72 & 0.79 \\
\hline Gender & & & 0.11 \\
\hline Male & 26 & 25 & \\
\hline Female & 2 & 7 & \\
\hline Race & & & 0.56 \\
\hline Hispanic & 21 & 20 & \\
\hline Caucasian & 6 & 5 & \\
\hline African American & 1 & 3 & \\
\hline Asian Pacific Islander & 1 & 3 & \\
\hline Etiology of Cirrhosis & & & \\
\hline Alcohol & 25 & 29 & 0.86 \\
\hline Hepatitis B & 6 & 10 & 0.20 \\
\hline Hepatitis C & 14 & 12 & 0.38 \\
\hline Alcohol and Hepatitis C & 7 & 4 & 0.21 \\
\hline NASH & 6 & 3 & 0.19 \\
\hline
\end{tabular}

Table 2. Site of Infection

\begin{tabular}{|c|c|}
\hline Trunk & 6 \\
\hline Lower Extremity & 17 \\
\hline Upper Extremity & 3 \\
\hline Scrotum & 2 \\
\hline
\end{tabular}

\section{Risk Factors}

Predisposing risk factors such as evidence or history of trauma, admission and discharge body mass index, tinea pedis, Human Immunodeficiency Virus (HIV) status, diabetes, homelessness, years of alcohol use and number of prior decompensations were compared (Table 3). Portal of entry was identified or history of blunt trauma was obtained in 17 of the 28 cellulitis cases. Documentation of tinea pedis was not available in most of the charts. A significant difference was found in admission body mass index, BMI, and homelessness.

Table 3. Risk Factors

\begin{tabular}{|l|c|c|c|}
\hline & Cellulitis & Control & p-Value \\
\hline \hline History/evidence of trauma & 17 & n/a & \\
\hline Diabetes & 6 & 3 & 0.17 \\
\hline HIV & 3 & 0 & 0.06 \\
\hline Years of Alcohol use & 22.5 & 18.4 & 0.12 \\
\hline Prior Decompensations & 2.2 & 1.8 & 0.15 \\
\hline Homeless & 13 & 4 & 0.004 \\
\hline Admission BMI & 28.9 & 26.3 & 0.04 \\
\hline Discharge BMI & 26.1 & 23.8 & 0.07 \\
\hline
\end{tabular}

HIV= Human Immunodeficiency Virus; BMI= Body Mass Index.

\section{Laboratory Data}

Comparison of admission laboratory data was extensive and did not yield a significant difference between the two groups (Table 4).

Table 4. Admission Laboratory Data

\begin{tabular}{|l|c|c|c|}
\hline & Cellulitis & Control & p-Value \\
\hline \hline Leukocytes & 8.5 & 8.6 & 0.81 \\
\hline Hemoglobin & 10.9 & 10.9 & 0.84 \\
\hline Platelet & 141.4 & 146.4 & 0.39 \\
\hline Sodium & 134 & 133 & 0.65 \\
\hline Blood Urea Nitrogen & 19.8 & 17.3 & 0.53 \\
\hline Creatinine & 0.98 & 1.1 & 0.65 \\
\hline Protime & 16.3 & 17.3 & 0.40 \\
\hline International Normalized Ratio & 1.56 & 1.67 & 0.42 \\
\hline Total Bilirubin & 4.7 & 6.4 & 0.46 \\
\hline Albumin & 2.13 & 2.15 & 0.82 \\
\hline Ascitic fluid total protein & 1.44 & 1.66 & 0.31 \\
\hline Serum Ascites-Albumin Gradient & 1.54 & 1.49 & 0.48 \\
\hline
\end{tabular}

\section{Objective Assessment}

Objective assessments upon admission included maximum temperature, altered mental status, body mass index, Child-Pugh score, icterus, degree of edema and presence of erythema. Degree of ascites and edema was significantly higher in the cellulitis group.

Twenty-seven patients with cellulitis had noticeable erythema.

\section{Subjective Assessment}

Seventeen of the 28 cellulitis patients complained of pain at the site of infection. 


\section{Wound and Blood Cultures}

Wound cultures were performed on three cellulitis patients. One patient's culture was negative but one patient grew Enterobacter cloacae and one Pseudomonas respectively. Blood cultures were negative in 5 out of 5 patients with cellulitis.

\section{Treatment}

Cellulitis patients received a mean of 11.9 days of intravenous antibiotic treatment (Table 6). Fourteen patients with cellulitis were treated with more than one class of antibiotic during their hospital course. First-generation cephalosporins were the most frequently used (Table 7). Diuretic treatment was greater in patients with cellulitis. Although patients with cellulitis required a greater number of days on higher dose diuretics, this difference was not significant. Hospital stay was greater by 4 days in the cellulitis patients compared to controls but this difference was not found to be significant.

Table 5. Admission Clinical Assessment

\begin{tabular}{|l|c|c|c|}
\hline & Cellulitis & Control & p-Value \\
\hline \hline Temperature & 99.9 & 99.9 & 0.90 \\
\hline Altered Mental Status & 12 & 10 & 0.35 \\
\hline Body Mass Index & 28.9 & 26.3 & 0.04 \\
\hline Child Pugh Score & 10.1 & 10.0 & 0.68 \\
\hline Icterus & 22 & 18 & 0.07 \\
\hline Degree of ascites & 2.3 & 1.5 & 0.03 \\
\hline Degree of edema & 2.1 & 1.5 & 0.04 \\
\hline
\end{tabular}

Table 6. Duration of Antibiotic and Diuretic Treatment and Length of Hospitalization

\begin{tabular}{|l|c|c|c|}
\hline \multicolumn{1}{|c|}{ Days of } & Cellulitis & Control & p-Value \\
\hline \hline Intravenous antibiotic treatment & 11.9 & N/A & N/A \\
\hline Oral antibiotic treatment & 4.1 & N/A & \\
\hline Diuretic treatment & 17.1 & 11.3 & 0.03 \\
\hline Higher Dose Diuretic & 7.3 & 4.4 & 0.15 \\
\hline Intravenous treatment & 11.9 & 3.9 & 0.0003 \\
\hline Length of Hospitalization & 22.3 & 18.3 & 0.26 \\
\hline
\end{tabular}

Intravenous treatment: includes antibiotic and diuretic treatment.

Higher Dose Diuretic: Furosemide dose greater than $40 \mathrm{mg} / \mathrm{d}$ and/or Spironolactone dose greater than $100 \mathrm{mg} / \mathrm{d}$.

\section{DISCUSSION}

Although cellulitis is a relatively common disorder, its exact etiology is unknown [13]. It is particularly important to identify risk factors in patients with cirrhosis to prevent recurrent hospitalizations. Although lymphedema is recognized as a risk factor for recurrent cellulitis [14], we believe that edema in the setting of cirrhosis should be considered as well.

Several studies have shown that the BMI is a risk factor for developing cellulitis $[15,16]$. We noted that admission body mass index was significantly higher in the cellulitis patients compared to controls at 28.9 and 26.3. At time of discharge, the BMI was higher in the cellulitis group compared to control group. This difference approached but did not reach our de- fined level of statistical significance. Higher doses of diuretics were used more frequently in the cellulitis group although there was not a statistically significant difference. It is well known that peripheral edema is a risk factor for cellulitis [17]. Increased edema or fluid retention results in increased patient weight. If there is an increased risk in cellulitis in cirrhotics, it is unclear if this reflects the degree of edema, increased dry weight, or both.

Table 7. Selection of Antibiotic Treatment

\begin{tabular}{|l|c|}
\hline \multicolumn{1}{|c|}{ Antibiotic } & Number of Patients * \\
\hline \hline First-Generation Cephalosporin & 17 \\
\hline Second-Generation Cephalosporin & 1 \\
\hline Third-Generation Cephalosporin & 5 \\
\hline Fourth-Generation Cephalosporin & 1 \\
\hline First-Generation Penicillin & 0 \\
\hline Second-Generation Penicillin & 3 \\
\hline Third-Generation Penicillin & 6 \\
\hline Fourth-Generation Penicillin & 4 \\
\hline First-Generation Fluoroquinolone & 0 \\
\hline Second-Generation Fluoroquinolone & 0 \\
\hline Third-Generation Fluoroquinolone & 6 \\
\hline Fourth-Generation Fluoroquinolone & 0 \\
\hline Cleocin & 1 \\
\hline Vancomycin & 4 \\
\hline Aminoglycoside & 1 \\
\hline Metronidazole & 4 \\
\hline *Includes patients who were on more than one antibiotic.
\end{tabular}

Tinea pedis has been shown to be a risk factor for cellulitis [14]. Although we considered tinea pedis as a risk factor, this risk factor was not consistently recorded in our chart review preventing our analysis.

Homelessness was a significant risk factor present in $46 \%$ of our cellulitis group compared to $12.5 \%$ of our controls. This is most likely reflective of a combination of risk factors which would include lack of general hygiene, greater risk for trauma and poor medical care.

Diabetes, hepatitis B and C infection, and HIV were not found to be significant risk factors in our study. Previous studies have identified diabetes as a risk factor $[19,20]$ while a recent case review did not [14]. Our review may have been too small to provide enough power to detect a difference. While our review was not able to identify hepatitis $\mathrm{C}$ viral infection as a risk factor, a much larger case control series comparing $34,204 \mathrm{HCV}$ infected patients with 136,816 control subjects found a significantly higher incidence of cellulitis amongst the HCV population [21].

Although an increased risk of bacteremia has been seen in a higher Child-Pugh class, our study did not the Child-Pugh score to be a significant variable [22].

A diagnosis of cellulitis based on objective assessment of laboratory data, blood cultures and temperature may be complicated in patients with cirrhosis where the hospital presentation is often in the setting of alcoholic hepatitis. Although a prior study identified hypoalbuminemia and renal insufficiency 
as risk factors for prolonged hospitalizations in patients with cellulitis, our study did not identify significant differences in laboratory variables between the two groups [23]. The maximum temperature was the same in the cellulitis and control group. Apyrexial presentation of cellulitis has been noted in other studies [12] and does not appear to be a reliable method to confirm the diagnosis of cellulitis. Blood cultures were negative in the 5 patients with cellulitis cases tested.

It has been documented that wound cultures are not helpful in the diagnosis of cellulites $[24,25]$. As the organism in the normal host tends to be gram-positive, contamination compounded by poor aspirate yield, makes the procedure less helpful. Corredoira et al. have suggested that in patients with cirrhosis, wound cultures may be helpful in identifying an unexpected gram-negative organism infection [1] Wound cultures were collected from three cellulitis patients. Wound culture was negative in one patient but the other two cultures yielded Enterobacter cloacae and Pseudomonas. As patients with cirrhosis are subject to recurrent hospitalizations, an additional consideration would be the increased risk of colonization by a methicillin resistant pathogens such as MRSA [26]. In the setting of cirrhosis, wound cultures may be helpful in identifying an infection by a gram-negative organisms or MRSA.

First-generation cephalosporins and third-generation penicillins were the most commonly selected first-line antibiotic treatment. Cephalexin and ampicillin-sulbactam were the most common choice in each class. Both generations provide gramnegative coverage. However, in patients who appeared to have a delayed resolution of signs and symptoms of cellulitis, antibiotic treatment was usually changed to fluoroquinolones. The average length of intravenous antibiotic treatment was 11.9 days. This duration of treatment appears quite long when compared to a 5.6 day treatment course in recent study of 332 hospitalized patients with cellulitis [23]. Whether it is necessary or appropriate to change from intravenous to oral treatment after this clinical resolution occurs is unclear. Intravenous antibiotic therapy may be prolonged leading to a longer hospital stay.

In summary, in the current era, cellulitis appears to be more common than SBP. An increase in awareness of this complication of advanced cirrhosis may lead to early treatment and improved outcomes. As the duration of hospitalization and cost of antibiotics are major factors in cost of treatment in hospitalized patients with cellulitis, further delineation of optimal treatment in patients with cirrhosis and cellulitis is warranted [26].

\section{REFERENCES}

[1] Bisno AL, Stevens DL. Streptococcal infections of skin and soft tissues. N Engl J Med 1996; 334: 240-5.

[2] Holzapfel L, Jacquet-Francillon T, Rahmani J, et al. Microbiological evaluation of infected wounds of the extremities in 214 adults. J Accid Emerg Med 1999; 16: 32-4.
[3] Yoon TY, Jung SK, Chang SH. Cellulitis due to Escherichia coli in three immunocompromised subjects. Br J Dermatol 1998; 139: 885-8.

[4] Neu HC. Infections due to gram-negative bacteria: an overview. Rev Infect Dis 1985; (7 Suppl 4): S778-82.

[5] Asmar BI, Bashour BN, Fleischmann LE. Escherichia coli cellulitis in children with idiopathic nephrotic syndrome. Clin Pediatr 1987; 26: 592-4.

[6] Olivier V, Desruelles F, Cua E, et al. Cellulite infectieuse recidivante et metastatique a Escherichia coli. Presse Med 2000; 29: 19-20.

[7] Arnold M, Woo ML, French GL. Vibrio vulnificus septicaemia presenting as spontaneous necrotising cellulitis in a woman with hepatic cirrhosis. Scand J Infect Dis 1989; 21: 727-31.

[8] Izumikawa K, Hazama H, Hara K, Miyazaki Y, Kamihira S, Kohno S. A case of a sudden death from Vibrio vulnificus septicemia in a patient with liver cirrhosis. Kansenshogaku Zasshi 1999; 73: 1159-62.

[9] Fernandez JM, Serrano M, De Arriba JJ, Sanchez MV, Escribano E, Ferreras P. Bacteremic cellulitis caused by Non-01, Non-0139 Vibrio cholerae: report of a case in a patient with hemochromatosis. Diagn Microbiol Infect Dis 2000; 37: 77-80.

[10] Ichiyama S, Hirai S, Minami T, et al. Campylobacter fetus subspecies fetus cellulitis associated with bacteremia in debilitated hosts. [comment]. Clin Infect Dis 1998; 27: 252-5.

[11] Corredoira JM, Ariza J, Pallares R, et al. Gram-negative bacillary cellulitis in patients with hepatic cirrhosis. Eur J Clin Microbiol Infect Dis 1994; 13: 19-24.

[12] Cox NH. Management of lower leg cellulitis. Clin Med 2002; 2: 23-7.

[13] Baddour LM. Cellulitis syndromes: an update. Int $\mathrm{J}$ Antimicrob Agents 2000; 14: 113-6.

[14] Dupuy A, Benchikhi H, Roujeau JC, et al. Risk factors for erysipelas of the leg (cellulitis): case-control study. BMJ 1999; 318: 1591-4.

[15] Lewis SD, Peter GS, Gómez-Marín O, Bisno AL. Risk Factors for Recurrent Lower Extremity Cellulitis in a U.S. Veterans Medical Center Population. Am J Med Sci 2006; 332(6): 304-07.

[16] Thorsteinsdottir B, Tleyjeh IM, Baddour LM. Abdominal wall cellulitis in the morbidly obese. Scand J Infect Dis 2005; 37(8): 605-08.

[17] Swartz M. Cellulitis. N Engl J Med 2004; 350: 904-12.

[18] Cox NH, Colver GB, Paterson WD. Management and morbidity of cellulitis of the leg. J R Soc Med 1998; 91: 634-7.

[19] Jorup-Ronstrom C. Epidemiological, bacteriological and complicating features of erysipelas. Scand J Infect Dis 1986; 18: 519-24.

[20] Eriksson B, Jorup-Ronstrom C, Karkkonen K, Sjoblom AC, Holm SE. Erysipelas: clinical and bacteriologic spectrum and serological aspects. Clin Infect Dis 1996; 23: 1091-8.

[21] El-Serag HB, Anand B, Richardson P, Rabeneck L. Association between hepatitis $\mathrm{C}$ infection and other infectious diseases: a case for targeted screening? Am J Gastroenterol 2003; 98: 167-74.

[22] Kuo CH, Changchien CS, Yang CY, Sheen IS, Liaw YF. Bacteremia in patients with cirrhosis of the liver. Liver 1991; 11: 334-9.

[23] Carratala J, Roson B, Fernandez-Sabe N, et al. Factors associated with complications and mortality in adult patients hospitalized for infectious cellulitis. Eur J Clin Microbiol Infect Dis 2003; 22: 151-7.

[24] Perl B, Gottehrer NP, Raveh D, Schlesinger Y, Rudensky B, Yinnon AM. Cost-effectiveness of blood cultures for adult patients with cellulitis. Clin Infect Dis 1999; 29: 1483-8.

[25] Sigurdsson AF, Gudmundsson S. The etiology of bacterial cellulitis as determined by fine-needle aspiration. Scand J Infect Dis 1989; 21: $537-42$.

[26] Vinken AG, Li JZ, Balan DA, Rittenhouse BE, Willke RJ, Goodman C. Comparison of linezolid with oxacillin or vancomycin in the empiric treatment of cellulitis in US hospitals. Am J Ther 2003; 10: 26474. 